\title{
Existence and multiplicity of solutions for nonlocal fourth-order elliptic equations with combined nonlinearities
}

Ru Yuanfang ${ }^{1 *}$ and An Yukun ${ }^{2}$

\section{"Correspondence:}

ruyanfangmm@163.com

${ }^{1}$ College of Science, China

Pharmaceutical University, 211198

Nanjing, P.R. China

Full list of author information is

available at the end of the article

\section{Abstract}

This paper is concerned with the following nonlocal fourth-order elliptic problem:

$$
\left\{\begin{array}{l}
\Delta^{2} u-m\left(\int_{\Omega}|\nabla u|^{2} d x\right) \Delta u=a(x)|u|^{s-2} u+f(x, u), \quad x \in \Omega, \\
u=\Delta u=0, \quad x \in \partial \Omega
\end{array}\right.
$$

by using the mountain pass theorem, the least action principle, and the Ekeland variational principle, the existence and multiplicity results are obtained.

Keywords: Fourth-order elliptic equation; Nonlocal; Asymptotically linear; Mountain pass theorem; Critical point

\section{Introduction}

In this paper, we consider the following nonlocal fourth-order elliptic problem:

$$
\left\{\begin{array}{l}
\Delta^{2} u-m\left(\int_{\Omega}|\nabla u|^{2} d x\right) \Delta u=a(x)|u|^{s-2} u+f(x, u), \quad x \in \Omega, \\
u=\Delta u=0, \quad x \in \partial \Omega
\end{array}\right.
$$

where $\Omega \subset R^{N}(N>4)$ is a bounded smooth domain, $m(\cdot) \in C\left(R^{+}, R^{+}\right), a(\cdot) \in C\left(\bar{\Omega}, R^{+}\right)$, $s \in(1,2)$, and $f \in C(\bar{\Omega} \times R, R)$.

Problem (1.1) is related to the stationary problems associated with

$$
\frac{\partial^{2} u}{\partial t^{2}}+\Delta^{2} u+\left(Q+\int_{\Omega}|\nabla u|^{2} d x\right) \Delta u=f\left(x, u, u_{t}\right)
$$

This plate model was proposed by Berger [1] in 1955, as a simplification of the von Karman plate equation which describes large defection of a plate, where the parameter $Q$ describes in-plane forces applied to the plate and the function $f$ represents transverse loads which may depend on the displacement $u$ and the velocity $u_{t}$.

(c) The Author(s) 2020. This article is licensed under a Creative Commons Attribution 4.0 International License, which permits use, sharing, adaptation, distribution and reproduction in any medium or format, as long as you give appropriate credit to the original author(s) and the source, provide a link to the Creative Commons licence, and indicate if changes were made. The images or other third party material in this article are included in the article's Creative Commons licence, unless indicated otherwise in a credit line to the material. If material is not included in the article's Creative Commons licence and your intended use is not permitted by statutory regulation or exceeds the permitted use, you will need to obtain permission directly from the copyright holder. To view a copy of this licence, visit http://creativecommons.org/licenses/by/4.0/. 
Because of the important background, several researchers have considered problem (1.1) by using variational methods when $a(x) \equiv 0$,

$$
\left\{\begin{array}{l}
\Delta^{2} u-m\left(\int_{\Omega}|\nabla u|^{2} d x\right) \Delta u=f(x, u), \quad x \in \Omega \\
u=\Delta u=0, \quad x \in \partial \Omega
\end{array}\right.
$$

with the function $m$ being bounded or unbounded and $f$ having superlinear growth. We refer the readers to [2-11] and the references therein.

Recently, in [12], Ru et al. considered problem (1.1) with $m(t)=a+b t$ and a more general $f$ such as

$$
\left\{\begin{array}{l}
\Delta^{2} u-\left(a+b \int_{\Omega}|\nabla u|^{2} d x\right) \Delta u=f(x, u, \nabla u, \Delta u), \quad x \in \Omega, \\
u=\Delta u=0, \quad x \in \partial \Omega .
\end{array}\right.
$$

By using an iterative method based on the mountain pass lemma and truncation method developed by De Figueiredo et al. [13], they proved that the above problem has at least one nontrivial solution.

One of the important conditions in their work is that $f(x, t)$ satisfies the famous Ambrosetti-Rabinowitz type condition, for short, which is called the (AR) condition:

(AR condition) there exist $\Theta>2$ and $t_{1}>0$, such that

$$
0<\Theta F\left(x, t, \xi_{1}, \xi_{2}\right) \leq t f\left(x, t, \xi_{1}, \xi_{2}\right), \quad \forall|t| \geq t_{1}, x \in \Omega,\left(\xi_{1}, \xi_{2}\right) \in R^{N+1}
$$

where $F\left(x, t, \xi_{1}, \xi_{2}\right)=\int_{0}^{t} f\left(x, s, \xi_{1}, \xi_{2}\right) d s$.

It is well known that (AR) is a important technical condition to apply the mountain pass theorem. This condition implies that

$$
\lim _{u \rightarrow \infty} \frac{F(x, u)}{u^{2}}=\infty
$$

If $f(x, u)$ is asymptotically linear at $u=0$ or $u=+\infty$. then $f(x, u)$ does not satisfy the (AR) condition. In [14], A. Bensedik and M. Bouchekif considered second-order elliptic equations of Kirchhoff type with an asymptotically linear potential

$$
\left\{\begin{array}{l}
-m\left(\int_{\Omega}|\nabla u|^{2} d x\right) \Delta u=f(x, u), \quad x \in \Omega \\
u=0, \quad x \in \partial \Omega
\end{array}\right.
$$

On the other hand, the classical equation involving a biharmonic operator

$$
\left\{\begin{array}{l}
\Delta^{2} u+c \Delta u=a(x)|u|^{s-2} u+f(x, u), \quad x \in \Omega, \\
u(x)=\Delta u(x)=0, \quad x \in \partial \Omega
\end{array}\right.
$$

has been extensively studied using the mountain pass theorem when $a(x) \equiv 0$ and $f(x, u)$ is asymptotically linear at $u=0$ or $u=+\infty$. We refer the reader to $[15,16]$. In particular, in [17], Pu et al. considered problem (1.2) when $a(x) \neq 0$. 
Until now, there are few works on problem (1.1) when $a(x) \neq 0$ and $f(x, u)$ does not satisfy the (AR) condition. Inspired by these references, in this paper, we discuss the existence and multiplicity of solutions of problem (1.1) when $a(x) \neq 0$ and the nonlinearity $f$ is asymptotically linear at $u=0$ or $u=+\infty$.

\section{Preliminaries}

Assume that the function $m(t)$ satisfies the following conditions:

(M) $m: R^{+} \rightarrow R^{+}$is continuous, nondecreasing, and there exists $m_{1} \geq m_{0}>0$ such that

$$
m_{0}=\min _{t \in R^{+}} m(t)=m(0), \quad m_{1}=\sup _{t \in R^{+}} m(t)
$$

Remark In [14] and [18], the function $m(t)$ is assumed that satisfy $(M)$ and there exits $t_{0}>0$ such that $m(t)=m_{1}, \forall t>t_{0}$.

First, we study the nonlinear eigenvalue problem

$$
\left\{\begin{array}{l}
\Delta^{2} u-m\left(\int_{\Omega}|\nabla u|^{2} d x\right) \Delta u=\Lambda u, \quad x \in \Omega, \\
u=0, \quad \Delta u=0, \quad x \in \partial \Omega .
\end{array}\right.
$$

Let $\left(\lambda_{k}, \phi_{k}\right)$ be the eigenvalue and the corresponding eigenfunction of $\left(-\Delta, H_{0}^{1}(\Omega)\right)$, namely

$$
\left\{\begin{array}{l}
-\Delta \phi_{k}=\lambda_{k} \phi_{k}, \quad x \in \Omega \\
\phi_{k}(x)=0, \quad x \in \partial \Omega
\end{array}\right.
$$

Set

$$
L u=\Delta^{2} u-m\left(\int_{\Omega}|\nabla u|^{2} d x\right) \Delta u
$$

Via some simple computations, we get

$$
\begin{aligned}
L \phi_{k} & =\Delta^{2} \phi_{k}-m\left(\int_{\Omega}\left|\nabla \phi_{k}\right|^{2} d x\right) \Delta \phi_{k} \\
& =\left[\lambda_{k}^{2}+\lambda_{k} m\left(\int_{\Omega}\left|\nabla \phi_{k}\right|^{2} d x\right)\right] \phi_{k} \\
& =\left[\lambda_{k}^{2}+\lambda_{k} m\left(\lambda_{k} \int_{\Omega}\left|\phi_{k}\right|^{2} d x\right)\right] \phi_{k} .
\end{aligned}
$$

Set

$$
\Lambda_{k}=\left\{\begin{array}{l}
\lambda_{k}^{2}+\lambda_{k} m\left(\int_{\Omega}\left|\nabla \phi_{k}\right|^{2} d x\right), \quad \text { or } \\
\lambda_{k}^{2}+\lambda_{k} m\left(\lambda_{k} \int_{\Omega}\left|\phi_{k}\right|^{2} d x\right)
\end{array}\right.
$$

and so $\Lambda_{k}(k=1,2, \ldots)$ are the eigenvalues of the operator $L$ associated to the eigenfunction $\phi_{k}$. 
Assume that the eigenfunctions $\phi_{k}$ are suitably normalized with respect to the $L^{2}(\Omega)$ inner product, namely

$$
\left(\phi_{i}, \phi_{j}\right)_{L^{2}(\Omega)}= \begin{cases}0, & i \neq j \\ 1, & i=j .\end{cases}
$$

Expression (2.1) can be rewritten as

$$
\Lambda_{k}=\lambda_{k}^{2}+\lambda_{k} m\left(\lambda_{k} \int_{\Omega}\left|\phi_{k}\right|^{2} d x\right)=\lambda_{k}^{2}+\lambda_{k} m\left(\lambda_{k}\right) .
$$

For each eigenvalue $\lambda_{k}$ being repeated as often as multiplicity, recall that

$$
0<\lambda_{1} \leq \lambda_{2} \leq \lambda_{3} \leq \cdots \leq \lambda_{k} \rightarrow+\infty,
$$

and if $(M)$ holds, then

$$
0<\Lambda_{1} \leq \Lambda_{2} \leq \Lambda_{3} \leq \cdots \leq \Lambda_{k} \rightarrow+\infty \text {. }
$$

Denote

$$
\bar{\Lambda}_{k}=\lambda_{k}^{2}+m_{1} \lambda_{k}, \quad k=1,2, \ldots,
$$

then we know that

$$
\Lambda_{k} \leq \bar{\Lambda}_{k}, \quad k=1,2, \ldots
$$

It is well known that

$$
\lambda_{1}=\inf \left\{\int_{\Omega}|\nabla u|^{2} d x: u \in \mathbf{H}_{0}^{1}(\Omega), \int_{\Omega}|u|^{2} d x=1\right\} .
$$

Similarly, we have

Lemma 2.1 Assume that (M) holds, then

$$
\begin{gathered}
\Lambda_{1}=\inf \left\{\int_{\Omega}|\Delta u|^{2} d x+m\left(\int_{\Omega}|\nabla u|^{2} d x\right) \int_{\Omega}|\nabla u|^{2} d x:\right. \\
\left.u \in \mathbf{H}^{2}(\Omega) \cap \mathbf{H}_{0}^{1}(\Omega), \int_{\Omega}|u|^{2} d x=1\right\} .
\end{gathered}
$$

Proof Denote

$$
\begin{gathered}
\inf \left\{\int_{\Omega}|\Delta u|^{2} d x+m\left(\int_{\Omega}|\nabla u|^{2} d x\right) \int_{\Omega}|\nabla u|^{2} d x:\right. \\
\left.u \in \mathbf{H}^{2}(\Omega) \cap \mathbf{H}_{0}^{1}(\Omega), \int_{\Omega}|u|^{2} d x=1\right\}=\Lambda_{0},
\end{gathered}
$$


then it is clear that

$$
\Lambda_{1}=\lambda_{1}^{2}+\lambda_{1} m\left(\lambda_{1}\right) \geq \Lambda_{0}
$$

Let $u_{0} \in \mathbf{H}^{2}(\Omega) \cap \mathbf{H}_{0}^{1}(\Omega)$ achieve $\Lambda_{0}$, then $\int_{\Omega}\left|u_{0}\right|^{2} d x=1, \int_{\Omega}\left|\nabla u_{0}\right|^{2} d x \geq \lambda_{1}$ and $u_{0}=0$ on $\partial \Omega$, therefore

$$
\int_{\Omega}\left|\nabla u_{0}\right|^{2} d x=-\int_{\Omega} u_{0} \Delta u_{0} d x
$$

which implies that

$$
\left(\int_{\Omega}\left|\nabla u_{0}\right|^{2} d x\right)^{2}=\left(-\int_{\Omega} u_{0} \Delta u_{0}\right)^{2} d x \leq \int_{\Omega}\left|u_{0}\right|^{2} d x \int_{\Omega}\left|\Delta u_{0}\right|^{2} d x=\int_{\Omega}\left|\Delta u_{0}\right|^{2} d x,
$$

then

$$
\begin{aligned}
\Lambda_{0} & =\int_{\Omega}\left|\Delta u_{0}\right|^{2} d x+m\left(\int_{\Omega}\left|\nabla u_{0}\right|^{2} d x\right) \int_{\Omega}\left|\nabla u_{0}\right|^{2} d x \\
& \geq\left(\int_{\Omega}\left|\nabla u_{0}\right|^{2} d x\right)^{2}+m\left(\int_{\Omega}\left|\nabla u_{0}\right|^{2} d x\right) \int_{\Omega}\left|\nabla u_{0}\right|^{2} d x \\
& \geq \lambda_{1}^{2}+\lambda_{1} m\left(\lambda_{1}\right)=\Lambda_{1} .
\end{aligned}
$$

So $\Lambda_{0}=\Lambda_{1}$.

Let $\mathbf{H}=\mathbf{H}^{2}(\Omega) \cap \mathbf{H}_{0}^{1}(\Omega)$ be the Hilbert space equipped with the standard inner product

$$
(u, v)_{H}=\int_{\Omega}(\Delta u \Delta v+\nabla u \nabla v) d x
$$

and the deduced norm

$$
\|u\|_{H}^{2}=\int_{\Omega}|\Delta u|^{2} d x+\int_{\Omega}|\nabla u|^{2} d x
$$

It is well know that $\|u\|_{H}$ is equivalent to $\left(\int_{\Omega}|\Delta u|^{2} d x\right)^{\frac{1}{2}}$. And there exists $\tau>0$ such that

$$
\int_{\Omega}|\Delta u|^{2} d x \leq\|u\|_{H}^{2} \leq \tau \int_{\Omega}|\Delta u|^{2} d x
$$

Denote

$$
\|u\|^{2}=\int_{\Omega}|\Delta u|^{2} d x+m_{1} \int_{\Omega}|\nabla u|^{2} d x
$$

and

$$
\|u\|_{m_{0}}^{2}=\int_{\Omega}|\Delta u|^{2} d x+m_{0} \int_{\Omega}|\nabla u|^{2} d x .
$$

It is obvious that the norms $\|u\|$ and $\|u\|_{m_{0}}$ are equivalent to the norm $\|u\|_{H}$ in $\mathbf{H}$. And since $m_{0}<m_{1}$, we have

$$
\|u\|^{2} \geq\|u\|_{m_{0}}^{2} \geq \theta\|u\|^{2}
$$

where $\theta=\frac{m_{0}}{m_{1}} \in(0.1)$. 
Throughout this paper, we denote by $C$ universal positive constants, unless otherwise specified, and

$$
\begin{aligned}
& \|u\|_{\infty}=\|u\|_{L^{\infty}} \quad \text { for } u \in \mathbf{L}^{\infty}(\Omega) \text { or } u \in \mathbf{C}(\bar{\Omega}), \\
& \|u\|_{q}=\left(\int_{\Omega}|\nabla u|^{q} d x\right)^{\frac{1}{q}} \text { for } u \in \mathbf{L}^{q}, 1 \leq q<+\infty .
\end{aligned}
$$

By the Sobolev embedding theorem, there is a positive $K_{q}$ such that

$$
\|u\|_{q} \leq K_{q}\|u\| \quad \text { for } u \in \mathbf{H} \text { and } 1 \leq q<\frac{2 N}{N-4} .
$$

Specially, when condition $(M)$ holds and $q=2$, by Lemma 2.1 , then

$$
\|u\|_{2}^{2} \leq \frac{1}{\Lambda_{1}}\|u\|^{2}
$$

The mountain pass theorem and the Ekeland variational principle are our main tools, which can be found in [19].

Lemma 2.2 Let E be a real Banach space, and $I \in C^{1}(E, R)$ satisfy $(P S)$ condition. Suppose

1 There exist $\rho>0, \alpha>0$ such that

$$
\left.I\right|_{\partial B_{\rho}} \geq I(0)+\alpha,
$$

where $B_{\rho}=\{u \in E \mid\|u\| \leq \rho\}$.

2 There is an $e \in E$ with $\|e\|>\rho$ such that

$$
I(e) \leq I(0) .
$$

Then I(u) has a critical value $c$ which can be characterized as

$$
c=\inf _{\gamma \in \Gamma} \max _{u \in \gamma([0,1])} I(u),
$$

where $\Gamma=\{\gamma \in C([0,1], E) \mid \gamma(0)=0, \gamma(1)=e\}$.

Lemma 2.3 Let $V$ be a complete metric space and $I: V \rightarrow R \cup\{+\infty\}$ be lower semicontinuous, bounded from below. Let $\varepsilon>0$ be given and $v \in V$ be such that

$$
I(v) \leq \inf _{V} I+\varepsilon .
$$

Then there exists $u \in V$ such that

$$
I(u) \leq I(v), \quad d(v, u) \leq 1
$$

and for all $w \neq u$ in $V$

$$
I(w)>F(u)-\varepsilon d(v, w) .
$$




\section{Main results}

A function $u \in \mathbf{H}$ is called a weak solution of (1.1) if

$$
\int_{\Omega} \Delta u \Delta v d x+m\left(\int_{\Omega}|\nabla u|^{2} d x\right) \int_{\Omega} \nabla u \nabla v d x-\int_{\Omega} a(x)|u|^{s-2} u v d x=\int_{\Omega} f(x, u) v d x
$$

holds for any $v \in \mathbf{H}$. Let $J: \mathbf{H} \rightarrow R$ be the functional defined by

$$
J(u)=\frac{1}{2} \int_{\Omega}|\Delta u|^{2} d x+\frac{1}{2} M\left(\int_{\Omega}|\nabla u|^{2} d x\right)-\frac{1}{s} \int_{\Omega} a(x)|u|^{s} d x-\int_{\Omega} F(x, u) d x
$$

where

$$
M(t)=\int_{0}^{t} m(s) d s, \quad F(t)=\int_{0}^{t} f(x, s) d s
$$

It is easy to see that $J \in C^{1}(\mathbf{H}, R)$ and the critical points of $J$ in $\mathbf{H}$ correspond to the weak solutions of problem (1.1).

We make the following assumptions.

(A) $a(x) \in \mathbf{C}(\bar{\Omega}), a(x) \geq 0, \forall x \in \bar{\Omega}$ and $\|a(x)\|_{\infty}=\bar{a}>0$;

$\left(F_{0}\right) t f(x, t) \geq 0$ for $x \in \bar{\Omega}, t \in \mathbf{R}$;

$\left(F_{1}\right) \lim _{|t| \rightarrow 0} \frac{f(x, t)}{t}=p(x)$ uniformly a.e. $x \in \Omega$, where $0<p(x) \in L^{\infty}(\Omega)$, and $\|p(x)\|_{\infty}<$ $\theta \Lambda_{1}$

$\left(F_{2}\right) \lim _{|t| \rightarrow+\infty} \frac{f(x, t)}{t}=l(-\infty<l<+\infty)$ uniformly a.e. $x \in \Omega$.

Our first main result is concluded as the following theorem:

Theorem 3.1 Assume the function $m(t)$ satisfies $(M), a(x)$ satisfies $(A)$, and the nonlinearity $f(x, t)$ satisfies $\left(F_{1}\right)$ and $\left(F_{2}\right)$, then problem (1.1) has at least one solution if $l<\Lambda_{1}$.

Proof It is easy to see, from condition $\left(F_{1}\right)$, that $f(x, 0)=0$ for $x \in \Omega$. So $u=0$ is the trivial solution of (1.1). From condition $\left(F_{2}\right)$, we can take $\varepsilon=\frac{1}{2}\left(\Lambda_{1}-l\right)>0$, and there exists $T>0$ such that

$$
f(x, t) t \leq(l+\varepsilon) t^{2}
$$

for all $|t| \geq T$ and a.e. $x \in \Omega$. By the continuity of $F$, there exists $C>0$ such that

$$
|F(x, t)| \leq \frac{l+\varepsilon}{2} t^{2}+C
$$

for all $(x, t) \in \Omega \times R$. On the other hand, from $(M)$ it follows that

$$
m_{0} t \leq M(t)=\int_{0}^{t} m(s) d s \leq m_{1} t, \quad \text { for } t>0
$$

Then we have

$$
\begin{aligned}
J(u) & =\frac{1}{2} \int_{\Omega}|\Delta u|^{2} d x+\frac{1}{2} M\left(\int_{\Omega}|\nabla u|^{2} d x\right)-\frac{1}{s} \int_{0}^{t} a(x)|u|^{s} d x-\int_{\Omega} F(x, u) d x \\
& \geq \frac{1}{2} \int_{\Omega}|\Delta u|^{2} d x+\frac{1}{2} m_{0} \int_{\Omega}|\nabla u|^{2} d x-\frac{1}{s} \bar{a} \int_{\Omega}|u|^{s} d x
\end{aligned}
$$




$$
\begin{aligned}
& -\frac{l+\varepsilon}{2} \int_{\Omega}|u|^{2} d x-C|\Omega| \\
\geq & \frac{1}{2}\|u\|^{2}-\frac{1}{s} K_{s} \bar{a}\|u\|^{s}-\frac{l+\varepsilon}{2 \Lambda_{1}}\|u\|^{2}-C|\Omega| \\
= & \frac{\Lambda_{1}-l-\varepsilon}{2 \Lambda_{1}}\|u\|^{2}-\frac{1}{s} K_{s} \bar{a}\|u\|^{s}-C|\Omega|,
\end{aligned}
$$

which shows that $J$ is coercive. Moreover, conditions $\left(F_{1}\right)$ and $\left(F_{2}\right)$ imply that $J$ is weakly lower semicontinuous in $\mathbf{H}$. Therefore we get a global minimum $u_{1}$ of $J$.

Next, we prove $u_{1} \neq 0$, so it is a nontrivial solution of (1.1). From condition $\left(F_{1}\right)$, there exists $C>0$ such that

$$
|f(x, t)| \leq C|t|
$$

for all $|t|$ small enough and $x \in \Omega$. It follows that

$$
|F(x, t)| \leq \frac{C}{2} t^{2}
$$

for all $|t|$ small enough and $x \in \Omega$. From condition $(A)$, we can chose $v \in \mathbf{H}$ such that

$$
\int_{\Omega} a(x)|v|^{s} d x>0
$$

Then we have

$$
\begin{aligned}
& \limsup _{t \rightarrow 0} \frac{J(t v)}{t^{s}} \\
& \quad=\limsup _{t \rightarrow 0} \frac{\frac{1}{2} \int_{\Omega}|\Delta(t v)|^{2} d x+\frac{1}{2} M\left(\int_{\Omega}|\nabla(t v)|^{2} d x\right)-\frac{1}{s} \int_{\Omega} a(x)|t v|^{s} d x-\int_{\Omega} F(x, t v) d x}{t^{s}} \\
& \quad \leq \limsup _{t \rightarrow 0} \frac{\frac{1}{2} \int_{\Omega}|\Delta(t v)|^{2} d x+\frac{1}{2} m_{1}\left(\int_{\Omega}|\nabla(t v)|^{2} d x\right)-\frac{1}{s} \int_{\Omega} a(x)|t v|^{s} d x-\int_{\Omega} F(x, t v) d x}{t^{s}} \\
& \leq \limsup _{t \rightarrow 0}\left(\frac{t^{2-s}}{2}\|v\|^{2}-\frac{1}{s} \int_{\Omega} a(x)|v|^{s} d x+\frac{C t^{2-s}}{2} \int_{\Omega} v^{2} d x\right) \\
& <0 .
\end{aligned}
$$

Therefore, we get that $J\left(u_{1}\right)<0$. It is clear that $J(0)=0$. Thus, $u_{1}$ is a nontrivial solution of (1.1).

Our second result is the following theorem:

Theorem 3.2 Assume the function $m(t)$ satisfies $(M), a(x)$ satisfies $(A)$, and the nonlinearity $f(x, t)$ satisfies $\left(F_{0}\right),\left(F_{1}\right)$, and $\left(F_{2}\right)$, then there exists a positive constant $a_{0}$ such that problem (1.1) has at least three nontrivial solutions if $\bar{a}<a_{0}$ and $\bar{\Lambda}_{1}<l<+\infty$.

Before proving Theorem 3.2, we give two lemmas.

Lemma 3.1 Suppose the conditions of Theorem 3.2 hold, then there exists a positive constant $a_{0}$ such that $J$ satisfies the following conditions for $\bar{a}<a_{0}$ and $\bar{\Lambda}_{1}<l<+\infty$ : 
Yuanfang and Yukun Boundary Value Problems

(2020) 2020:130

Page 9 of 16

1. There exist constants $\rho>0, \alpha>0$ such that $\left.J\right|_{\partial B_{\rho}} \geq \alpha$ with $B_{\rho}=\{u \in \mathbf{H}:\|u\| \leq \rho\}$;

2. $J\left(t \varphi_{1}\right) \rightarrow-\infty$ as $t \rightarrow+\infty$.

Proof (Claim 1) By $\left(F_{1}\right)$ and $\left(F_{2}\right)$, there exists $C>0$ such that for all $(x, t) \in \Omega \times R$ and $p \in\left(1, \frac{N+4}{N-4}\right)$, we have

$$
F(x, t) \leq \frac{1}{4}\left(\|p(x)\|_{\infty}+\theta \Lambda_{1}\right) t^{2}+C|t|^{p+1} .
$$

From inequalities (2.2), (2.3) and (3.1), we have

$$
\begin{aligned}
J(u)= & \frac{1}{2} \int_{\Omega}|\Delta u|^{2} d x+\frac{1}{2} m\left(\int_{\Omega}|\nabla u|^{2} d x\right)-\frac{1}{s} \int_{0}^{t} a(x)|u|^{s} d x-\int_{\Omega} F(x, u) d x \\
\geq & \frac{1}{2} \int_{\Omega}|\Delta u|^{2} d x+\frac{1}{2} m_{0} \int_{\Omega}|\nabla u|^{2} d x-\frac{1}{s} \bar{a} \int_{\Omega}|u|^{s} d x \\
& -\frac{1}{4}\left(\|p(x)\|_{\infty}+\theta \Lambda_{1}\right)\|u\|_{2}^{2}-C\|u\|_{p+1}^{p+1} \\
\geq & \frac{\theta}{2}\|u\|^{2}-\frac{1}{s} \bar{a} K_{s}\|u\|^{s}-\frac{1}{4} \frac{\left(\|p(x)\|_{\infty}+\theta \Lambda_{1}\right)}{\Lambda_{1}}\|u\|^{2}-C K_{p+1}\|u\|^{p+1} \\
= & \left(\frac{\theta \Lambda_{1}-\|p(x)\|_{\infty}}{4 \Lambda_{1}}-\frac{1}{s} \bar{a} K_{s}\|u\|^{s-2}-C K_{p+1}\|u\|^{p-1}\right)\|u\|^{2} .
\end{aligned}
$$

Setting

$$
a_{0}=\frac{s}{2 K_{s} K_{p+1}^{\frac{2-s}{p-1}}}\left(\frac{\theta \Lambda_{1}-\|p(x)\|_{\infty}}{8 \Lambda_{1}}\right)^{\frac{p-s+1}{p-1}}, \quad \rho=\left(\frac{\theta \Lambda_{1}-\|p(x)\|_{\infty}}{8 \Lambda_{1} C K_{p+1}}\right)^{\frac{1}{p-1}}
$$

when $\bar{a} \leq a_{0}$ and $\|u\|=\rho$, it follows that

$$
J(u) \geq\left(\frac{\theta \Lambda_{1}-\|p(x)\|_{\infty}}{16 \Lambda_{1}}\right)\|\rho\|^{2}=\alpha>0 .
$$

So, Claim 1 is proved.

(Claim 2) By $\left(F_{2}\right)$ and for $l>\bar{\Lambda}_{1}$, there exists $C>0$ such that

$$
F(x, t) \geq \frac{1}{4}\left(l+\bar{\Lambda}_{1}\right) t^{2}-C
$$

for all $(x, t) \in \Omega \times R$. Let $\lambda_{1}$ and $\phi_{1}$ be the first eigenvalue and eigenfunction of $\left(-\Delta, H_{0}^{1}(\Omega)\right)$ with $\int_{\Omega}\left|\phi_{1}\right|^{2} d x=1$. We know that

$$
\bar{\Lambda}_{1}=\int_{\Omega}\left|\Delta \phi_{1}\right|^{2} d x+m_{1} \int_{\Omega}\left|\nabla \phi_{1}\right|^{2} d x=\lambda_{1}^{2}+m_{1} \lambda_{1} .
$$

Then, we have

$$
\begin{aligned}
J\left(t \phi_{1}\right)= & \frac{1}{2} \int_{\Omega}\left|\Delta\left(t \phi_{1}\right)\right|^{2} d x+\frac{1}{2} m\left(\int_{\Omega}\left|\nabla\left(t \phi_{1}\right)\right|^{2} d x\right) \\
& -\frac{1}{s} \int_{\Omega} a(x)\left|t \phi_{1}\right|^{s} d x-\int_{\Omega} F\left(x, t \phi_{1}\right) d x
\end{aligned}
$$




$$
\begin{aligned}
\leq & \frac{t^{2}}{2} \int_{\Omega}\left|\Delta \phi_{1}\right|^{2} d x+\frac{t^{2}}{2} m_{1} \int_{\Omega}\left|\nabla \phi_{1}\right|^{2} d x \\
& -\frac{t^{s}}{s} \int_{\Omega} a(x)\left|\phi_{1}\right|^{s} d x-\frac{t^{2}}{4}\left(l+\bar{\Lambda}_{1}\right) \int_{\Omega}\left|\phi_{1}\right|^{2} d x+C|\Omega| \\
= & \frac{t^{2}}{4}\left(\bar{\Lambda}_{1}-l\right)-\frac{t^{s}}{s} \int_{\Omega} a(x)\left|\phi_{1}\right|^{s} d x+C|\Omega| .
\end{aligned}
$$

Hence, $J\left(t \psi_{1}\right) \rightarrow-\infty, t \rightarrow+\infty$.

The proof of Lemma 3.1 is completed.

Let

$$
f^{+}(x, t)= \begin{cases}f(x, t), & t \geq 0 \\ 0, & t<0\end{cases}
$$

and

$$
f^{-}(x, t)= \begin{cases}f(x, t), & t \leq 0 \\ 0, & t>0\end{cases}
$$

Define functionals $J^{ \pm}: \mathbf{H} \rightarrow \mathbf{R}$ as follows:

$$
J^{ \pm}(u)=\frac{1}{2} \int_{\Omega}|\Delta u|^{2} d x+\frac{1}{2} m\left(\int_{\Omega}|\nabla u|^{2} d x\right)-\frac{1}{s} \int_{\Omega} a(x)|u|^{s} d x-\int_{\Omega} F^{ \pm}(x, u) d x,
$$

where $F^{ \pm}(t)=\int_{0}^{t} f^{ \pm}(x, s) d s$.

Lemma 3.2 Assume that $(M),(A)$ and $\left(F_{0}\right)-\left(F_{2}\right)$ hold, and $\bar{\Lambda}_{1}<l<+\infty$, then $J^{ \pm}(u)$ satisfies the $(P S)$ condition.

Proof We just prove that $J^{+}(u)$ satisfies the (PS) condition. The proof for $J^{-}(u)$ is similar. Let $\left\{u_{n}\right\} \in \mathbf{H}$ be a (PS) sequence, namely

$$
\begin{aligned}
& J^{+}\left(u_{n}\right) \rightarrow c, \\
& \nabla J^{+}\left(u_{n}\right) \rightarrow 0 .
\end{aligned}
$$

Firstly, we claim that $\left\{u_{n}\right\}$ is bounded in $\mathbf{H}$. If not, we may assume that $\left\|u_{n}\right\| \rightarrow+\infty$ as $n \rightarrow+\infty$. Let $w_{n}=\frac{u_{n}}{\left\|u_{n}\right\|}$, then $\left\|w_{n}\right\|=1$. Passing to a subsequence, we may assume that there exists $w \in \mathbf{H}$ such that

$$
\begin{cases}w_{n} \rightarrow w & \text { in } \mathbf{H} \\ w_{n} \rightarrow w & \text { in } \mathbf{L}^{r}(\Omega), 1 \leq r \leq \frac{2 N}{N-4} \\ w_{n} \rightarrow w & \text { a.e. in } \Omega\end{cases}
$$

By $\left(F_{1}\right)$ and $\left(F_{2}\right)$, we see that there exist $C_{1}$ and $C_{2}$ such that

$$
\left|\frac{f(x, t)}{t}\right| \leq C_{1}, \quad\left|\frac{F(x, t)}{t^{2}}\right| \leq C_{2}
$$


for all $(x, t) \in \Omega \times \mathbf{R}$ and define

$$
\left.\frac{f(x, t)}{t}\right|_{t=0}=\lim _{t \rightarrow 0} \frac{f(x, t)}{t},\left.\quad \frac{F(x, t)}{t^{2}}\right|_{t=0}=\lim _{t \rightarrow 0} \frac{F(x, t)}{t^{2}} .
$$

Then we claim that $w \neq 0$. Otherwise, if $w \equiv 0$, we know that $w_{n} \rightarrow 0$ strongly in $\mathbf{L}^{r}(\Omega)$. Dividing (3.2) by $\left\|u_{n}\right\|^{2}$, we have

$$
\begin{aligned}
\frac{J^{+}\left(u_{n}\right)}{\left\|u_{n}\right\|^{2}}= & \frac{1}{2\left\|u_{n}\right\|^{2}}\left(\int_{\Omega}\left|\Delta u_{n}\right|^{2} d x+m\left(\int_{\Omega}\left|\nabla u_{n}\right|^{2} d x\right)\right) \\
& -\frac{1}{s\left\|u_{n}\right\|^{2-s}} \int_{\Omega} a(x)\left|w_{n}(x)\right|^{s} d x-\int_{\Omega} \frac{F^{+}\left(x, u_{n}\right)}{\left\|u_{n}\right\|^{2}} d x \\
= & o(1) .
\end{aligned}
$$

It follows from (3.1) and (3.5) that

$$
\begin{aligned}
\frac{\theta}{2} & \leq \frac{1}{2\left\|u_{n}\right\|^{2}}\left(\int_{\Omega}\left|\Delta u_{n}\right|^{2} d x+m_{0} \int_{\Omega}\left|\nabla u_{n}\right|^{2} d x\right) \\
& \leq \frac{1}{2\left\|u_{n}\right\|^{2}}\left(\int_{\Omega}\left|\Delta u_{n}\right|^{2} d x+m\left(\int_{\Omega}\left|\nabla u_{n}\right|^{2} d x\right)\right) \\
& =\frac{1}{s\left\|u_{n}\right\|^{2-s}} \int_{\Omega} a(x)\left|w_{n}(x)\right|^{s} d x+\int_{\Omega} \frac{F^{+}\left(x, u_{n}\right)}{\left\|u_{n}\right\|^{2}} d x+o(1) \\
& \leq \frac{\bar{a}}{s\left\|u_{n}\right\|^{2-s}} \int_{\Omega}\left|w_{n}(x)\right|^{s} d x+C_{2} \int_{\Omega}\left|w_{n}(x)\right|^{2} d x+o(1) \rightarrow 0,
\end{aligned}
$$

which is impossible, so $w \neq 0$.

Let us define

$$
\Omega_{0}=\{x \in \Omega \mid w(x)=0\}, \quad \Omega_{1}=\{x \in \Omega \mid w(x) \neq 0\} .
$$

Then, for all $v \in \mathbf{H}$, we have

$$
\begin{aligned}
\left|\int_{\Omega_{0}} \frac{f^{+}\left(x, u_{n}\right)}{u_{n}} w_{n} v d x\right| & \leq C_{1} \int_{\Omega_{0}}\left|w_{n}\right||v| d x \\
& \leq C_{1}\left(\int_{\Omega_{0}}\left|w_{n}\right|^{2} d x\right)^{\frac{1}{2}}\left(\int_{\Omega_{0}}|v|^{2} d x\right)^{\frac{1}{2}} .
\end{aligned}
$$

So,

$$
\lim _{n \rightarrow+\infty} \int_{\Omega_{0}} \frac{f^{+}\left(x, u_{n}\right)}{u_{n}} w_{n} v d x=0=\int_{\Omega_{0}} l w^{+} v d x
$$

where $w^{+}(x)=\max \{w(x), 0\}$. On the other hand, since $\left\|u_{n}\right\| \rightarrow+\infty$, we have $\left|u_{n}(x)\right|=$ $\left\|u_{n}\right\|\left|w_{n}(x)\right| \rightarrow+\infty$ for $x \in \Omega_{1}$. Therefore, by $\left(F_{2}\right)$ and the dominated convergence theorem, we get

$$
\lim _{n \rightarrow+\infty} \int_{\Omega_{1}} \frac{f^{+}\left(x, u_{n}\right)}{u_{n}} w_{n} v d x=\int_{\Omega_{1}} \lim _{n \rightarrow+\infty} \frac{f^{+}\left(x, u_{n}\right)}{u_{n}} w_{n} v d x=\int_{\Omega_{1}} l w^{+} v d x
$$


Combining (3.6) and (3.7), we obtain

$$
\lim _{n \rightarrow+\infty} \int_{\Omega} \frac{f^{+}\left(x, u_{n}\right)}{u_{n}} w_{n} v d x=\int_{\Omega} l w^{+} v d x
$$

Now, (3.3) implies that, for all $v \in \mathbf{H}$, we have

$$
\begin{aligned}
\left(\nabla J^{+}\left(u_{n}\right), v\right)= & \int_{\Omega} \Delta u_{n} \Delta v d x+m\left(\int_{\Omega}\left|\nabla u_{n}\right|^{2} d x\right) \int_{\Omega} \nabla u_{n} \nabla v d x \\
& -\int_{\Omega} a(x)\left|u_{n}(x)\right|^{s-1} v d x-\int_{\Omega} f^{+}\left(x, u_{n}\right) v d x \rightarrow 0 .
\end{aligned}
$$

Dividing by $\left\|u_{n}\right\|$, we get

$$
\begin{aligned}
& \int_{\Omega} \Delta w_{n} \Delta v d x+m\left(\int_{\Omega}\left|\nabla u_{n}\right|^{2} d x\right) \int_{\Omega} \nabla w_{n} \nabla v d x \\
& -\frac{1}{\left\|u_{n}\right\|^{2-s}} \int_{\Omega} a(x)\left|w_{n}(x)\right|^{s-1} v d x-\int_{\Omega} \frac{f^{+}\left(x, u_{n}\right)}{u_{n}} w_{n} v d x \rightarrow 0 .
\end{aligned}
$$

Since

$$
\left\|u_{n}\right\|^{2}=\int_{\Omega}\left|\Delta u_{n}\right|^{2} d x+m_{1} \int_{\Omega}\left|\nabla u_{n}\right|^{2} d x \rightarrow+\infty
$$

as $n \rightarrow+\infty$, we can suppose that there exists a subsequence, still denoted $\left\{\int_{\Omega}\left|\nabla u_{n}\right|^{2} d x\right\}$, such that

$$
\int_{\Omega}\left|\nabla u_{n}\right|^{2} d x \rightarrow+\infty, \quad n \rightarrow+\infty
$$

otherwise, there exists $K>0$ such that

$$
\int_{\Omega}\left|\nabla u_{n}\right|^{2} d x \leq K
$$

and furthermore, there exist a subsequence, still denoted $\left\{\int_{\Omega}\left|\nabla u_{n}\right|^{2} d x\right\}$, and a constant $t^{\prime} \geq 0$ such that

$$
\int_{\Omega}\left|\nabla u_{n}\right|^{2} d x \rightarrow t^{\prime}, \quad n \rightarrow+\infty
$$

In case (3.10) holds, by $(M)$, we have

$$
\lim _{n \rightarrow+\infty} m\left(\int_{\Omega}\left|\nabla u_{n}\right|^{2} d x\right)=m_{1}
$$

Combining (3.4), (3.8), (3.9) and (3.10), as $n \rightarrow+\infty$, we obtain

$$
\int_{\Omega} \Delta w \Delta v d x+m_{1} \int_{\Omega} \nabla w \nabla v d x=\int_{\Omega} l w^{+} v d x, \quad \forall v \in \mathbf{H}
$$


Taking $v=\phi_{1}$ in (3.13), we have

$$
\int_{\Omega} \Delta w \Delta \phi_{1} d x+m_{1} \int_{\Omega} \nabla w \nabla \phi_{1} d x=\int_{\Omega} l w^{+} \phi_{1} d x
$$

Noticing that $\phi_{1}$ is the positive solution of

$$
\left\{\begin{array}{l}
\Delta^{2} u+m_{1} \Delta u=\bar{\Lambda}_{1} u, \quad \text { in } \Omega \\
u=0, \quad \Delta u=0, \quad \text { on } \partial \Omega
\end{array}\right.
$$

we have

$$
\int_{\Omega} \Delta w \Delta \phi_{1} d x+m_{1} \int_{\Omega} \nabla w \nabla \phi_{1} d x=\int_{\Omega} \bar{\Lambda}_{1} w \phi_{1} d x
$$

Thus, from (3.14) and (3.15), we get

$$
\int_{\Omega} l w^{+} \phi_{1} d x=\int_{\Omega} \bar{\Lambda}_{1} w \phi_{1} d x
$$

If $w(x) \geq 0$ a.e. in $\Omega$, since $w(x) \neq 0$, we have $\int_{\Omega} w \phi_{1} d x>0$. Then (3.15) implies that

$$
\int_{\Omega} l w \phi_{1} d x=\int_{\Omega} l w^{+} \phi_{1} d x=\int_{\Omega} \bar{\Lambda}_{1} w \phi_{1} d x
$$

which contradicts $l>\bar{\Lambda}_{1}$. Otherwise, let $\Omega_{-}=\{x \in \Omega \mid w(x)<0\}$ and suppose $\left|\Omega_{-}\right|>0$. Then $\int_{\Omega_{-}}-w \phi_{1} d x>0$ and $\int_{\Omega} w^{+} \phi_{1} d x>\int_{\Omega} w \phi_{1} d x>0$. It follows from (3.15) again that

$$
\int_{\Omega} l w^{+} \phi_{1} d x=\int_{\Omega} \bar{\Lambda}_{1} w \phi_{1} d x<\int_{\Omega} \bar{\Lambda}_{1} w^{+} \phi_{1} d x
$$

which contradicts $l>\bar{\Lambda}_{1}$.

So $\left\{u_{n}\right\}$ is bounded in $\mathbf{X}$.

In case (3.11) holds, by $(M)$, we have

$$
\lim _{n \rightarrow+\infty} m\left(\int_{\Omega}\left|\nabla u_{n}\right|^{2} d x\right)=m\left(t^{\prime}\right)=m^{\prime} \leq m_{1} .
$$

Combining (3.4), (3.8), (3.9) and (3.17), as $n \rightarrow+\infty$, we obtain

$$
\int_{\Omega} \Delta w \Delta v d x+m^{\prime} \int_{\Omega} \nabla w \nabla v d x=\int_{\Omega} l w^{+} v d x, \quad \forall v \in \mathbf{H} .
$$

Taking $v=\phi_{1}$ in (3.18), we have

$$
\int_{\Omega} \Delta w \Delta \phi_{1} d x+m^{\prime} \int_{\Omega} \nabla w \nabla \phi_{1} d x=\int_{\Omega} l w^{+} \phi_{1} d x
$$

Notice that $\phi_{1}$ is also the positive solution of

$$
\left\{\begin{array}{l}
\Delta^{2} u+m^{\prime} \Delta u=\Lambda_{1}^{\prime} u, \quad \text { in } \Omega, \\
u=0, \quad \Delta u=0, \quad \text { on } \partial \Omega,
\end{array}\right.
$$


where $\Lambda_{1}^{\prime}=\lambda_{1}^{2}+m^{\prime} \lambda_{1}$. Then we have

$$
\int_{\Omega} \Delta w \Delta \phi_{1} d x+m^{\prime} \int_{\Omega} \nabla w \nabla \phi_{1} d x=\int_{\Omega} \Lambda_{1}^{\prime} w \phi_{1} d x
$$

From (3.19) and (3.20), we get

$$
\int_{\Omega} l w^{+} \phi_{1} d x=\int_{\Omega} \Lambda_{1}^{\prime} w \phi_{1} d x
$$

Notice that for $\Lambda_{1}^{\prime} \leq \bar{\Lambda}_{1}$, similar to the discussions in case (3.10) holds, (3.21) implies a contradiction to $l>\bar{\Lambda}_{1}$.

So $\left\{u_{n}\right\}$ is bounded in $\mathbf{X}$.

Now, since $\Omega$ is bounded and $\left(F_{1}\right),\left(F_{2}\right)$ hold, by using the Sobolev embedding theorem and the standard procedures, we can easily prove that $\left\{u_{n}\right\}$ has a convergent subsequence. The proof of the lemma is completed.

Proof of Theorem 3.2. From the proof of Lemma 3.1, it is easy to see that $J^{+}(u)$ and $J^{-}(u)$ satisfy the conditions of Lemma 3.1. So there exist $\rho>0, \alpha>0$, and $e \in \mathbf{H}$ with $\|e\|>\rho$ such that

$$
\left.J^{ \pm}(u)\right|_{\partial B_{\rho}} \geq \alpha>0, \quad J^{ \pm}(e)<0 .
$$

It is clear that $J^{ \pm}(0)=0$. Moreover, by Lemma 3.2, the functionals $J^{ \pm}$satisfy the (PS) condition. By Lemma 2.2, we know that $J^{ \pm}$has the critical value $c^{ \pm}$, respectively, which can be characterized as

$$
c^{ \pm}=\inf _{\gamma \in \Gamma} \max _{u \in \gamma([0,1])} J^{ \pm}(u)
$$

where $\Gamma=\{\gamma \in C([0,1], \mathbf{H}) \mid \gamma(0)=0, \gamma(1)=e\}$. So there exist critical points $u_{1}, u_{2} \in \mathbf{H}$ such that

$$
J^{+}\left(u_{1}\right)=c^{+}>0, \quad J^{-}\left(u_{2}\right)=c^{-}>0 .
$$

Since $f^{+}(x, t) \geq 0$ and $f^{-}(x, t) \leq 0$, by the comparison principles for some fourth order elliptic problems [20], $u_{1}$ is a positive solution of (1.1) and $u_{2}$ is a negative solution of (1.1).

Next, we prove that problem (1.1) has another solution $u_{3} \in \mathbf{H}$ such that $J\left(u_{3}\right)<0$. For $\rho>0$ given by Lemma 3.1, define $B_{\rho}=\{u \in E:\|u\| \leq \rho\}$ and then $B_{\rho}$ is a complete metric space with the distance $\operatorname{dist}(u, v)=\|u-v\|$ for $u, v \in B_{\rho}$. By Lemma 3.1, we know that

$$
\left.J(u)\right|_{\partial B_{\rho}} \geq \alpha>0
$$

Clearly, $J \in C^{1}\left(B_{\rho}, R\right)$, so $J$ is bounded from below on $B_{\rho}$. And we know that $J$ is lower semicontinuous.

Similar to the proof of Theorem 3.1, there exists $v \in \mathbf{H}$ such that

$$
\lim _{t \rightarrow 0} \frac{J(t v)}{t^{p}}<0
$$


Then letting $c_{1}=\inf \left\{J(u): u \in B_{\rho}\right\}$, we get that $c_{1}<0$. By Lemma 2.3, for any $k>0$, there is a $\left\{u_{k}\right\}$ such that

$$
c_{1} \leq J\left(u_{k}\right) \leq c_{1}+\frac{1}{k}
$$

Now we claim that $\left\|u_{k}\right\|<\rho$ for $k$ large enough. Otherwise, if $\left\|u_{k}\right\|=\rho$ for infinitely many $k$, and, without loss of generality, we may suppose that $\left\|u_{k}\right\|=\rho$ for all $k>1$. It follows from (3.22) that $J\left(u_{k}\right) \geq \alpha>0$. Letting $k \rightarrow \infty$, we see that $0>c_{1} \geq \alpha>0$, which is a contradiction.

For any $u \in E$ with $\|u\|=1$, let

$$
w_{k}=u_{k}+t u
$$

for any fixed $k \geq 1$. We get

$$
\left\|w_{k}\right\| \leq\left\|u_{k}\right\|+t
$$

so $w_{k} \in B_{\rho}$ for $t>0$ small enough. It follows from Lemma 2.3 that

$$
J\left(w_{k}\right)=J\left(u_{k}+t u\right) \geq J\left(u_{k}\right)-\frac{t}{k}\|u\| .
$$

Thus, we have

$$
J^{\prime}\left(u_{k}\right)=\lim _{t \rightarrow 0^{+}} \frac{J\left(u_{k}+t u\right)-J\left(u_{k}\right)}{t} \geq-\frac{1}{k}
$$

and

$$
J^{\prime}\left(u_{k}\right)=\lim _{t \rightarrow 0^{+}} \frac{J\left(u_{k}-t u\right)-J\left(u_{k}\right)}{t} \leq \frac{1}{k} .
$$

Then $\left|J^{\prime}\left(u_{k}\right)\right| \leq \frac{1}{k} \rightarrow 0$ and $J\left(u_{k}\right) \rightarrow c_{1}$ as $k \rightarrow \infty$. Therefore $\left\{u_{k}\right\}$ is a (PS) sequence at level $c_{1}$. From Lemma 3.2, $\left\{u_{k}\right\}$ has a convergent subsequence. Hence, we see that there exists $u_{3} \in \mathbf{H}$ such that $J^{\prime}\left(u_{3}\right)=0$ and $J\left(u_{3}\right)=c_{1}<0$. Thus, $u_{3}$ is a nontrivial weak solution of (1.1) and $u_{3} \neq u_{1}, u_{3} \neq u_{2}$.

\section{Acknowledgements}

We would like to thank the referee for his/her valuable comments and helpful suggestions which have led to an improvement of the presentation of this paper.

\section{Funding}

This work was supported by the Fundamental Research Funds for the Central Universities (2632020PY02) and the National Natural Foundation of China-NSAF (Grant No. 11571092).

\section{Availability of data and materials}

Not applicable.

\section{Competing interests}

The authors declare that they have no competing interests.

\section{Authors' contributions}

All the authors contributed equally and significantly in writing this article. All the authors read and approved the final manuscript. 


\section{Author details}

${ }^{1}$ College of Science, China Pharmaceutical University, 211198 Nanjing, P.R. China. ${ }^{2}$ College of Science, Nanjing University of Aeronautics and Astronautics, 210016 Nanjing, P.R. China.

\section{Publisher's Note}

Springer Nature remains neutral with regard to jurisdictional claims in published maps and institutional affiliations.

Received: 21 April 2020 Accepted: 24 July 2020 Published online: 31 July 2020

\section{References}

1. Berger, M.: A new approach to the large detection of plate. J. Appl. Mech. 22, 465-472 (1955)

2. Afrouzi, G.A., Moradi, S., Caristi, G.: Infinitely many solutions for impulsive nonlocal elastic beam equations. Differ. Equ. Dyn. Syst. (2017). https://doi.org/10.1007/s12591-017-0397-z

3. Ansari, H., Vaezpour, S.M., Hesaaraki, M.: Existence of positive solution for nonlocal singular fourth order Kirchhoff equation with Hardy potential. Positivity 21, 1545-1562 (2017)

4. Cabada, A., Figueiredo, G.M.: A generalization of an extensible beam equation with critical growth in RN. Nonlinear Anal., Real World Appl. 20, 134-142 (2014)

5. Ferrara, M., Khademloo, S., Heidarkhani, S.: Multiplicity results for perturbed fourth-order Kirchhoff type elliptic problems. Appl. Math. Comput. 234, 316-325 (2014)

6. Figueiredo, G.M., Nascimeto, R.G.: Multiplicity of solutions for equations involving a nonlocal term and biharmonic operator. Electron. J. Differ. Equ. 2016, 217 (2016)

7. Heidarkhani, S., Ferrara, M., Khademloo, S.: Nontrivial solutions for one-dimensional fourth-order Kirchhoff-type equations. Mediterr. J. Math. 13, 217-236 (2016)

8. Khoutir, S., Chen, H.: Ground state solutions and least energy sign-changing solutions for a class of fourth order Kirchhoff-type equations in $\mathbb{R}^{N}$. Arab J. Math. Sci. 23, 94-108 (2017)

9. Ma, T.F.: Positive solutions for a nonlinear Kirchhoff type beam equation. Appl. Math. Lett. 18, 479-482 (2005)

10. Ma, T.F., Martinez, A.L.M.: Positive solutions for a fourth order equation with nonlinear boundary conditions. Math. Comput. Simul. 80, 2177-2184 (2010)

11. Wang, F., An, T., An, Y.: Existence of solutions for fourth order elliptic equations of Kirchhoff type on $\mathbb{R}^{n}$. Electron. J. Qual. Theory Differ. Equ. 2014, 39 (2014)

12. Ru, Y., Wang, F., Wang, Y., An, T.: On fourth-order elliptic equations of Kirchhoff type with dependence on the gradient and the Laplacian. J. Funct. Spaces 2018, 9857038 (2018)

13. De Figueiredo, D., Girardi, M., Matzeu, M.: Semilinear elliptic equations with dependence on the gradient via mountain pass technique. Differ. Integral Equ. 17, 119-126 (2004)

14. Bensedik, A., Bouchekif, M.: On an elliptic equation of Kirchhoff type with a potential asymptotically linear at infinity. Math. Comput. Model. 49, 1089-1096 (2009)

15. An, Y., Liu, R.: Existence of nontrivial solutions of a asymptotically linear fourth-order elliptic equation. Nonlinear Anal. 68, 3325-3331 (2008)

16. Hu, S., Wang, L.: Existence of nontrivial solutions for fourth-order asymptotically linear elliptic equations. Nonlinear Anal. 94, 120-132 (2014)

17. Pu, Y., Wu, X., Tang, C.: Fourth-order Navier boundary value with combined nonlinearities. J. Math. Appl. 398, 798-813 (2013)

18. Wang, F., An, Y.: Existence and multiplicity of solutions for a fourth order elliptic equation. Bound. Value Probl. 2012, 6 (2012)

19. Ekeland, I.: Nonconvex minimization problems. Bull. Am. Math. Soc. (N.S.) 1(3), 443-474 (1979)

20. Goyal, V.B., Schaefer, P.W.: Comparison principles for some fourth order elliptic problems. Lect. Notes Math. 964, 272-279 (1982)

\section{Submit your manuscript to a SpringerOpen ${ }^{\circ}$ journal and benefit from:}

- Convenient online submission

- Rigorous peer review

- Open access: articles freely available online

- High visibility within the field

- Retaining the copyright to your article

Submit your next manuscript at $\gg$ springeropen.com 